\title{
Relationship between the morphology of A-1 segment of anterior cerebral artery and anterior communicating artery aneurysms
}

\author{
Wenfeng Feng, Long Zhang, Weiguang Li, Guozhong Zhang, Xiaoyan He, Gang Wang, Mingzhou Li, Songtao Qi*
}

Department of Neurosurgery, Nanfang Hospital, 1838 Guang zhou Dadao Road, Guangzhou 510515, China.

\begin{abstract}
:
Background: The anterior communicating artery $(\mathrm{ACOA})$ is one of the most frequent sites for cerebral aneurysm. The peculiar directions of projection of aneurysms offer great challenges to clinical treatment.

Objetives: To establish the relationship between morphology of A-1 segment of anterior cerebral artery (ACA) and aneurismal projection.

Methods: Randomly selected digital subtraction angiography data of 264 anterior communicating artery aneurysms (ACoAA) cases and 296 cases of other cerebral vascular diseases in the same period were retrospectively analyzed.

Results: Among 264 ACoAA patients, the morphology of A-1 segment showed type 1a in 158 sides, type 1b in 11, type 2a in 35, type $12 \mathrm{~b}$ in 87, type 3 in 171 and absence in 66. The morphology of A-1 segment in 296 patients with other cerebral vascular diseases displayed type $1 \mathrm{a}$ in 195 sides, type $1 \mathrm{~b}$ in 20, type $2 \mathrm{a}$ in 47, type $2 \mathrm{~b}$ in 74, type 3 in 217 and absence in 39. The non-visualization of A-1 segment in the group of ACoAA occurred more than in the control group $\left(\chi^{2}=11.482\right.$, $p=0.001)$. The classifications of ACoAAs in 264 patients were confirmed as anterior-superior type in 121 cases, anteriorinferior type in 105, complicated type in 16, posterior-inferior type in 12 and posterior-superior type in 10 . The correlation between morphology of A-1 segment of ACA and classifications of ACoAA was significant $(p=0.000 ; C=0.619, p=0.000)$. The direction of ACoAA was downward when the A-1 segment of ACA was Type 1a or Type 2a, and was upward when it was Type 1a or Type 2a and was upward or downward or complicated when it was Type 3.

Conclusion: The relationship between morphology of A-1 segment of ACA and classification of ACoAA is clarified in the present study, which is helpful to surgical treatment.
\end{abstract}

Key words: anterior cerebral artery; morphology, A-1 segment; anterior communicating artery, aneurysm African Health Sciences 2014;14(1): 83-88 http://dx.doi.org/10.4314/ahs.v14i1.13

\section{Introduction}

Anterior communicating artery aneurysm (ACoAA) has been regarded as the most complex among anterior circulation aneurysms. It takes up approximately $30 \%$ of all intracranial aneurysms, and gives rise to more severe morbidity and mortality ${ }^{1,2}$. Sequelae will be always present after clinical treatment ${ }^{3}$. Previous papers showed that the change of hemodynamics ${ }^{4}$. Cognitive deficits associated with this specific played an important role in the pathogenesis of ACoAA pathological change have been observed in patients treated with either coil embolization or surgicalclipping if ACoA mostly affects the hemodynamic circulation ${ }^{5,6}$.

Yasargil described ACoAA as anterior, superior,

$$
\begin{aligned}
& \text { Corresponding author: } \\
& \text { Songtao Qi, } \\
& \text { Department of Neurosurgery, } \\
& \text { Nanfang Hospital, 1838 Guang zhou Dadao Road } \\
& \text { Guangzhou 510515, China. } \\
& \text { E-mail: feng121218@gmail.com } \\
& \text { Tel: +020-61687231 }
\end{aligned}
$$

posterior, inferior and complex according to the projection of the aneurysmal body ${ }^{7}$, which evolved into anterior-inferior, anterior-superior, posteriorsuperior, posterior-inferior and complicated types. The morphology of proximal segment (A-1) segment of anterior cerebral artery (ACA) included "arc" pattern (Type 1a: convex shape; Type 1b: concave shape), "S" pattern (Type 2a: inverse lateral "S" shape; Type 2b: lateral "S" shape), approximate straight-line shape (Type 3) and non-development.

The formation of ACoAA has been reported to be correlated with the A-1 dominancy of the anterior cerebral artery (ACA). However, the morphology of A-1 segment of ACA and its relationship with the projection of ACoAA were rarely investigated ${ }^{8}$. The present study aimed to investigate the relationship between the morphology of A-1 segment and the formation and projection of ACoAA, in order to provide valuable reference for surgical plan.

\section{Methods \\ Patient population}

Selection criteria: 269 patients with ACoAA confirmed using digital subtraction angiography (DSA) and 300 
patients with other cerebrovascular diseases and who were seen from January 2005 to January 2012 in Southern Hospital Neurosurgery. Patients with the following symptoms were excluded as they may cause morphologic changes of A-1 segment: severe cerebral vasospasm determined by DSA and severe cerebral swelling or large intracranial hematoma indicated by cranial computerized tomography (CT) or MRI. A total of 9 patients were excluded including 5 ACoAA and 4 others. 147 males and 117 females consisted 264 cases of ACoAA with ages ranging from 22 to 75 and an average age of $49.02 \pm 10.09$. They were classified with Hunt-Hess grades when admitted: Grade $0, n=18$; Grade 1, $\mathrm{n}=84$; Grade 2, $\mathrm{n}=90$; Grade 3, $\mathrm{n}=48$; Grade 4, $\mathrm{n}=22$; Grade 5, $\mathrm{n}=2.161$ males and 135 females consisted 296 cases of other cerebrovascular diseases (135 cases of aneurysm at other intracranial sites, 107 cases with intracranial arteriovenous malformation, 28 cases with dural arteriovenous fistula and 26 cases with others like intracranial artery stenosis) aging from 6 to 82 with an average of $50.53 \pm 11.25$.

\section{DSA examination}

All cases were inserted with catheters into right femoral arteries with Seldinger technique, and subjected to bilateral internal, external carotid artery and vertebral arteriography in order to completely show the images of arterial phase, capillary phase, venous phase and sinus stage. They were at least subjected to standard right, lateral and oblique position imaging. Patients suspected of aneurysm, arteriovenous malformation or other lesions were underwent 3D rotational angiography. Diagnosis reports were reviewed by 3 doctors with different seniorities with 2 above associate director.

\section{Classification criteria for the morphology of $A-1$ segment of $A C A$}

According to DSA angiography from standard right and lateral position, combined with oblique position image and 3D rotational angiography, the morphology of A-1 segment was divided into several types following the anterior-interior to posterior-exterior direction. Type 1 is the "arc" pattern (Type 1a: convex shape; Type 1b: concave shape); Type 2 is the "S" pattern (Type 2a: inverse lateral "S" shape; Type 2b: lateral "S" shape); Type 3 is the approximate straight-line shape and non-development. With ACoAA, the morphology of A-1 segment from dominant image side was chosen to analyse the relationship with aneurysm projection. $1 \mathrm{a}$ and $2 \mathrm{a}, 1 \mathrm{~b}$ and $2 \mathrm{~b}$ were combined as inferior and superior types, respectively, according to inner blood flow direction for statistic analysis due to the few sample funds of $1 \mathrm{~b}$ and $2 \mathrm{a}$ (shown in Fig. 1).

Fig. 1 The morphology of left A1 segment of ACA

A: Concave shape; B: Convex shape; C: Lateral "S" shape; D: Inverse lateral "S" shape; E: Approximate straightline shape.

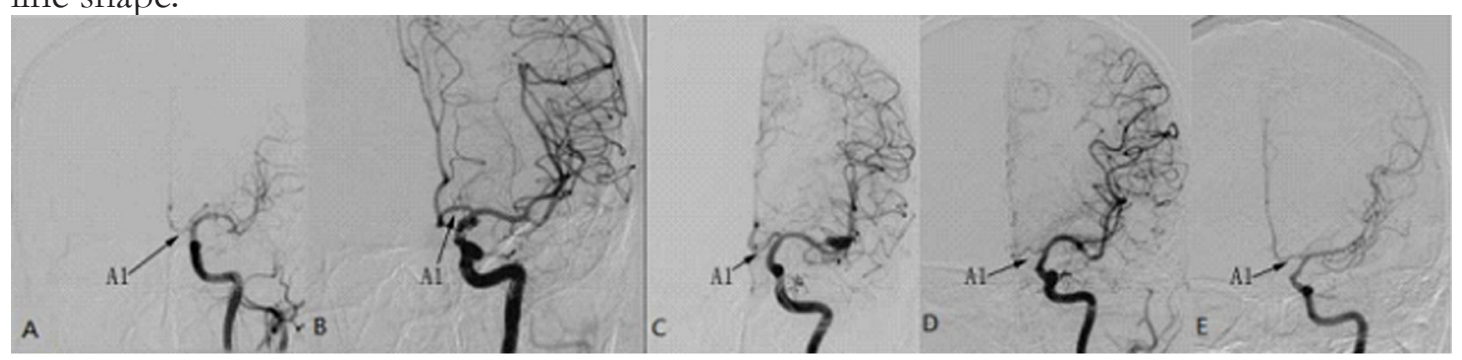

Fig. 1

Determination criteria for the projection of $A C 0 A A$ ${ }^{9}$ According to DSA angiography images from standard right and lateral position combined with $3 \mathrm{D}$ rotational angiography, rectangular coordinates were established taking the projection of aneurysm neck shown in the image of standard lateral position as the origin point. The surrounding of aneurysm neck was divided into four areas including anterior-inferior, anteriorsuperior, posterior-superior and posterior-inferior with the horizontal and vertical axis. Thus the types of aneurismal body projection were named as anteriorinferior, anterior-superior, posterior-superior, posteriorinferior and complicated types (shown in Fig. 2). 
Fig. 2 Classification of ACoAA in cerebral angiography

A: Complicated type; B: Anterior-superior type; C: Posterior-superior type; D: Posterior-inferior type; E: Anteriorinferior type.

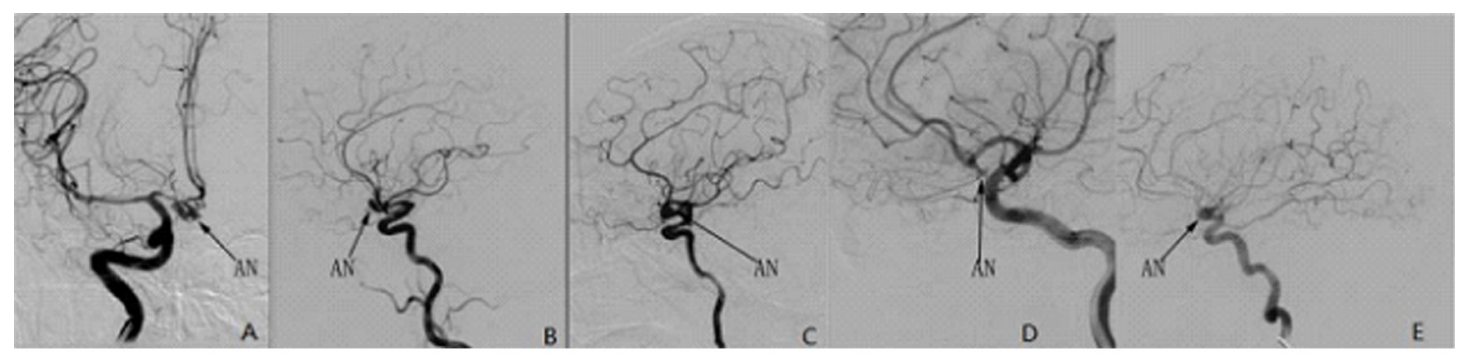

Fig. 2

\section{Statistical analysis}

SPSS 13.0 was used for the statistical analysis. Quantitative data fitted normal distribution were expressed with means $\underline{ \pm}$ sd and qualitative data were described using frequency and percentage. Quantitative data between groups were compared using Pearson chisquare test. Two-way disordered classification data were tested for their correlation using Likelihood Ratio test. Statistically significant level was considered as significant $\mathrm{p}<0.05$ and very significantly $\mathrm{p}<0.01$.

\section{Results}

Morphology comparison of $A-1$ segment of $A C A$

A-1 segment in the group with ACoAA was more frequently absent than the group with other cerebrovascular diseases $\left(\chi^{2}=11.482, P=0.001\right)$. The constituent ratios of two groups were in the same order as: $3>1 \mathrm{a}>2 \mathrm{~b}>2 \mathrm{a}>1 \mathrm{~b}$ with no significant difference $\left(\chi^{2}=6.645, p=0.156\right)$ (shown in Table 1$)$.

Table 1 Comparison of morphology of A1 segment of ACA of two groups

\begin{tabular}{cccccccc}
\hline \multirow{2}{*}{ Group } & Cases & \multicolumn{5}{c}{ Morphology of A1 segment of ACA [n (\%)] } \\
\cline { 3 - 8 } & $(\mathrm{n})$ & $1 \mathrm{a}$ & $1 \mathrm{~b}$ & $2 \mathrm{a}$ & $2 \mathrm{~b}$ & 3 & ND \\
\hline ACoAA & 528 & $158(29.9)$ & $11(2.1)$ & $35(6.6)$ & $87(16.5)$ & $171(32.4)$ & $66(12.5)$ \\
Others & 592 & $195(32.9)$ & $20(3.4)$ & $47(7.9)$ & $74(12.5)$ & $217(36.7)$ & $39(6.6)$ \\
\hline
\end{tabular}

ND: non-developed

The proportion of constituent ratio of $A C O A A$ projection

The proportion of constituent ratio of ACoAA projection was as following: 121 cases of anteriorsuperior type $(45.8 \%)>105$ cases of anterior-inferior type $(39.8 \%)>16$ cases of complicated type $(6.1 \%)>$ 12 cases of posterior-inferior type $(4.5 \%)>10$ cases of posterior-superior type $(3.8 \%)$.

The relationship between morphology of $A-1$ segment from dominant image and the projection of aneurysm

A significant correlation of the morphology of A-1 segment with aneurysm projection $\left(\chi^{2}=221.859, p<0.01\right)$ with Contingency Coefficient of $0.619(p<0.01)$. ACoAA generally pointed downward when A-1 segment was Type 1a or 2a and it pointed upward when A-1 segment was Type $1 \mathrm{~b}$ or $2 \mathrm{~b}$. When A-1 was Type 3 , the projection of ACoAA was any upward, downward or complicated (shown in Table 2 and 3). 
Table 2 Relationship between the morphology of A1 segment of ACA and the classification of ACoAA

\begin{tabular}{|c|c|c|c|c|c|c|}
\hline \multirow{2}{*}{$\begin{array}{l}\text { Morphology of } \\
\text { A-1 segment }\end{array}$} & \multirow{2}{*}{$\begin{array}{l}\text { Cases } \\
\text { (n) }\end{array}$} & \multicolumn{5}{|c|}{ Classification of ACoAA image $[\mathrm{n}(\%)]$} \\
\hline & & $\begin{array}{l}\text { Anterior- } \\
\text { inferior }\end{array}$ & $\begin{array}{l}\text { Anterior- } \\
\text { superior }\end{array}$ & $\begin{array}{l}\text { Posterior- } \\
\text { superior }\end{array}$ & $\begin{array}{l}\text { Posterior- } \\
\text { inferior }\end{array}$ & Complicated \\
\hline $1 \mathrm{a}$ & 87 & 73 (83.9) & & & $9(10.3)$ & $5(5.8)$ \\
\hline $1 b$ & 4 & & $2(50.0)$ & $2(50.0)$ & & \\
\hline $2 a$ & 5 & $2(40.0)$ & & & $3(60.0)$ & \\
\hline $2 \mathrm{~b}$ & 63 & & $55(87.3)$ & $3(4.8)$ & & $5(7.9)$ \\
\hline 3 & 105 & $30(28.6)$ & $64(61.0)$ & $5(4.8)$ & & $6(5.7)$ \\
\hline
\end{tabular}

Table 3 Relationship between the merged morphology of A1 segment of ACA and the merged classification of ACoAA

\begin{tabular}{ccccc}
\hline \multirow{2}{*}{ Morphology of A-1 segment } & Cases (n) & \multicolumn{3}{c}{ Classification of ACoAA image [n (\%)] } \\
\cline { 3 - 5 } & & Upward & Downward & Complicated \\
\hline Inner blood flow points to & 92 & $87(94.6)$ & $0(0.0)$ & $5(5.4)$ \\
$\begin{array}{c}\text { inferior } \\
\text { Inner blood flow points to } \\
\text { superior }\end{array}$ & 67 & $0(0.0)$ & $62(92.5)$ & $5(7.5)$ \\
Approximate straight-line shape & 105 & $30(28.6)$ & $69(65.7)$ & $6(5.7)$ \\
\hline
\end{tabular}

\section{Discussion}

The relationship of morphology of $A-1$ segment of $A C A$ with the formation of $A C O A A$

Variations of A-1 segment mainly come from imbalance development (the ratio of one side to the opposite caliber of A-1 segment is greater than or equal to 2), dysplasia (caliber of A-1 is smaller than $0.5 \mathrm{~mm}$ ) and absence. They caused hemodynamic changes in the circle of Willis which was very important to aneurysm ${ }^{10}$. Previous reports showed that variations of A-1 segment on one side increased the occurrence of aneurysm ${ }^{11}$. Non-development in DSA of A-1 segment on one side meant the absence or severe dysplasia on this side. In our study, the cases of non-development type of A-1 segment in ACoAA group were obviously more than control group. All data from DSA cerebral angiography indicated very few balance images of both right and left side of A-1 segment while one side image was more dominant than the opposite side, which suggested the correlation between variations (A-1 absence or severe dysplasia) and the formation of ACoAA.

A-1 segment originated from internal carotid artery in right angle, mostly with various tortuosities rather than straight forward to anterior-interior from posterior- exterior ${ }^{12}$. One is the "arc" pattern with a shorter A-1 segment. Most of this pattern is a convex shape which is stretched tightly over the chiasm. Few are concave shapes which related to the distance from the optic tract, chiasma or nerves. Another pattern is the " $\mathrm{S}$ " shape with a longer A-1 segment. Lateral " $\mathrm{S}$ " shape is the majority with inner part pointing superior-interior and outer part pointing inferior-exterior which are related to tightly adhere to optic tract, chiasma or nerves and the long gap between front and back chiasma, respectively; Inverse lateral "S" shape is uncommon with inner part pointing inferior-interior and outer part pointing superior-exterior. The downward direction of outer part is suggested to be related to the long distance of the origin of A-1 segment from the periphery of optic tract, chiasma or nerves and not adhere to the top while the upward direction of the inner part is related to adherence to the top of the optic tract, chiasma or nerves. The most popular pattern of A-1 segment is the approximate straight-line shape, showing straight or approximate linear from posterior-exterior to anteriorinterior. The study suggested that A-1 morphology patterns were commonly approximate linear, convex and lateral "S" shape while concave and inverse lateral "S" shape were rare. There were no significant differences 
for the constituent ratios of A-1 morphology between the ACoAA and the control group. The results suggested that, the formation of ACoAA might have no or little effect on the morphology of A-1segment which is possible to be resulted from the small and bias samples.

Relationship between the morphology of $A-1$ segment and the projection of $A C O A A$

A-1 segment of ACA displays various shapes and variations. The pressures of left and right side of A-1 are unequal, companied with increased blood flow and pressure, leading to the change of hemodynamics. Generally speaking, the junction of dominant A-1, ACA and the ipsilateral distal segment (A-2) has a significant change of hemodynamics with high wall shear stress (WSS) and it's the predilection site of ACoAA ${ }^{13}$. The present study showed that, the developing effect of aneurysm on one side was better than the opposite side, part of which was even not developed using internal carotid artery arteriography. Most of the aneurysms were at the juntion of dominant A-1, ACA and the ipsilateral A-2. Based on this study, there was a significant relationship between the morphology of dominant A-1 segment and the projection of aneurysm with a Contingency Coefficient bigger than 0.5.

Although with various tortuousness, upward, downward and horizontal were the only three directions of the inner blood flow of A-1 segment. When the A-1 segment appeared from exterior-superior to interiorinferior in approximate linear, convex or inverse lateral "S" shape, the inner blood flowed downwards which caused the downward projection of ACoAA. When A-1 appeared from exterior-inferior to interior-superior in approximate linear, concave or lateral "S" shape, the blood flowed upwards which caused the upward projection of ACoAA. When A-1 stretched straightly from exterior to interior, the blood flowed horizontally and the ACoAA generally projected to the opposite side. In most cases, the ACoAA pointed anterior for A-1 usually extended from posterior-exterior to anteriorinterior.

Clinical significance of the relationship between A-1 morphology and $A C o A A$ projection in medical treatment

When A-1 segment is undeveloped, it is very important to keep ACA patent when either surgical clipping or endovascular embolization is carried out in order to avoid the development of infarction.

The A-1 segment appeared from exterior-superior to interior-inferior in convex, inverse lateral " $\mathrm{S}$ " or approximate linear shape caused decrease of vertical distance between ACA Complex and anterior skull base 14. The aneurysm adhered tightly to skull base, optic chiasma and nerves, which would easily cause abnormal bleeding with excessive dragging during operation. Furthermore, the optic chiasma, nerves and feeding arteries would be crushed during aneurysm separation, leading to visual disturbances.

The A-1 segment appeared from exterior-inferior to interior-superior in concave, lateral " $\mathrm{S}$ " or approximate linear shape caused increase of vertical distance between ACA Complex and anterior skull base. The aneurysm might be partly or even wholly embedded into lateral or bilateral rectus gyri, which made it difficult to expose during operation. Sometimes, partial resection of rectus gyri was needed to expose the aneurysm, and it would be complicated with memory decrease and cognitive impairment.

When A-1 segment rarely appeared from anteriorexterior to posterior-interior and prefixed optic chiasm was obvious, the distance between posterior margin of anterior communicating artery and the endplate was shortened. Big aneurysm was highly related to endplate, and the feeding arteries, leading to difficult separation, hypothalamus and perforator vessel injuries, and severe postoperative reaction ${ }^{15}$.

During intravascular interventional treatment of ACoAA, shaping the microcatheter with an adequate angle and its durability are essential for a successful procedure of embolization of aneurysms ${ }^{16}$. Generally, the dominant A-1 segment was chosen as the vascular route during embolization procedure. When A-1 were "arc" or approximate straight-line shapes, the microcatheter was generally steam shaped to a single bending. The bending length depended on the size of the aneurysm and the diameter of parent artery, and curvature degree was based on the angle between aneurismal body and parent artery. When A-1 was "S" shape, the microcatheter was usually steam shaped to a double bending. This kind of bending mainly depended on the pattern of A-1 segment, and also the size of aneurysm, diameter of the parent artery and the angle between aneurismal body and parent artery. When A-1 segment originated from internal carotid artery in right angle with "S" shape, it's difficult for microcatheter to pass the internal carotid arterial bifurcation, so the micro-wire needed to be multi-shaped to guide the 
catheter into the right position.

Careful reading of the image of DSA cerebral angiography and making clear the presence of A-1 dominancy, the projection of ACoAA, the morphology of dominant A-1 segment and its relationship with aneurismal projection are clinically important for analyzing operative difficulties, preventing accidental injury during operation and reducing complications. Evaluation on the relationship between A-1 morphology and aneurismal projection was not systematically carried out for the small sample size in this study. With the improvement of sample size and multi-central research, detailed results will be got in the future.

\section{References:}

1. Andaluz N, Van Loveren HR, Keller JT, Zuccarello M. Anatomic and clinical study of the orbitopterional approach to anterior communicating artery aneurysms, Neurosurgery 2003; 52:1140-1149.

2. Kassell NF, Torner JC, Haley EC Jr, Jane JA, Adams HP, Kongable GL. The international cooperative study on the timing of aneurysm surgery: Part 1-Overall management results. J. Neurosurg. 1990; 73: 18-36.

3. DeLuca J, Diamond BJ. Aneurysm of the anterior communicating artery: A review of neuroanatomical and neuropsychological sequelae. J. Clin. Exp. Neuropsychol. 1995; 17: 100-121.

4. Hassan T, Hassan AA, Ahmed YM. Influence of parent vessel dominancy on fluid dynamics of anterior communicating artery aneurysms. Acta Neurochir. 2011; 153: 305-310.

5. Chan A, Ho S, Poon WS. Neuropsychological sequelae of patients treated with microsurgical clipping or endovascular embolization for anterior communicating artery aneurysm. Eur. Neurol. 2002; 47: 37-44.

6. Volpe BT, Hirst W. Amnesia following the rupture and repair of an anterior communicating artery aneurysm. J. Neurol. Neurosurg. Psychiatry. 1983;46(8):704-709.

7. Yasargil MG. Anterior cerebral and anterior communicating artery aneurysms. In: Yasargil MG (ed) Microneurosurgery vol II. Clinical considerations, surgery of the intracranial aneurysms and results. Thieme, New York, 1984; pp 165-231.
8. Weil AG, Bojanowski MW, Scholtes F, Darsaut TE, Signorelli F, Weill A. Angiographic Pitfall: Duplicated Tapered A1 Segment of the Anterior Cerebral Artery Mimicking an Anterior Communicating Artery Aneurysm. Interv. Neuroradiol. 2011;17:179182.

9. Li JP, Zhao JZ, Wang S, Zhao YL, Zhang D. Classification and surgical treatment of anterior communicating artery aneurysms, Chin. J. Clin. Neurosurg. 2008; 13(9): 514-515.

10. Hoi Y, Meng H, Woodward SH, Bendok BR, Hanel RA, Guterman LR, Hopkins LN. Effects of arterial geometry on aneurysm growth: three-dimensional computational fluid dynamics study, J. Neurosurg. 2004; 101: 676-681.

11. Sasaki T, Kodama N, Matsumoto M, Suzuki K, Konno Y, Sakuma J, Endo Y, Oinuma M. Blood flow disturbance in perforating arteries attributable to aneurysm surgery, J. Neurosurg. 2007; 107:60-67.

12. Perlmutter D, Rhoton AL Jr. Microsurgical anatomy of the anterior cerebral-anterior communicatingrecurrent artery complex, J. Neurosurg. 1976; 45: 259272.

13. Li C, Wang S, Chen J, Yu H, Zhang Y, Jiang F, $\mathrm{Mu} \mathrm{S}$, Li H, Yang X. Influence of hemodynamics on recanalization of totally occluded intracranial aneurysms: a patient-specific computational fluid dynamic simulation study. J. Neurosurg. 2012; 117: 276283.

14. Kang DH, Park J, Park SH, Hamm IS. Saccular aneurysm at the anterior communicating artery complex associated with an accessory middle cerebral artery: Report of two cases and review of the literature, J. Korean Neurosurg. Soc. 2009; 46: 568-571.

15. Debono B, Proust F, Langlois O, Clavier E, Douvrin F, Derrey S, Freger P. Ruptured anterior communicating artery aneurysm. Therapeutic options in 119 consecutive cases. Chin. J. Nerv. Ment. Dis. 2009; 35(9): 517-520.

16. Kiyosue H, Hori Y, Matsumoto S, Okahara M, Tanoue S, Sagara Y, Mori H. Shapability, memory, and luminal changes in microcatheters after steam shaping: a comparison of 11 different microcatheters. Am. J. Neuroradiol. 2005; 26: 2610-2616. 